\title{
BRAGG RESONANCES IN A TWO-LAYER FLUID
}

\author{
W. D. MCKEE ${ }^{1}$
}

(Received October 1993)

\begin{abstract}
Equations are derived to approximately describe the propagation of small amplitude surface and interfacial waves across small irregularities in depth in a two-layer fluid. When the irregularities are sinusoidal, Bragg interaction effects between an incident surface wave and the bottom corrugations can lead to a large-amplitude reflected interfacial wave or a large-amplitude transmitted interfacial wave if the incident surface wave is relatively long and the lower layer shallow in comparison with the upper layer.
\end{abstract}

\section{Introduction}

Over the past decade, the subject of Bragg reflection of water waves propagating across corrugated bottoms has received much attention from both a theoretical and an experimental point of view. Put simply, Bragg reflection in this context is an enhanced reflection which occurs when the wavenumber of an incident surface wave is approximately one half the wavenumber of the corrugations in the bottom. This phenomenon has attracted some interest in the coastal engineering community as a possible means of protection against wave damage. There is also the intriguing suggestion that the mass transport in the bottom boundary layer due to the partially standing nature of the wavetrain when Bragg reflection is operating may in some way be responsible for the growth and maintenance of the sandbars forming the corrugations in the bottom. The reader is referred to Mei [3], Mei, Hara and Naciri [4] and Kirby [1] for the theoretical background and to O'Hare and Davies [5] for some comparisons between theory and experiment.

This paper will consider wave propagation in a system consisting of two superposed fluids overlying a corrugated bottom. It will be assumed that the amplitudes of the bottom corrugations are small compared with the depth of the lower layer. This system supports two types of wave motion - surface and interfacial. In the former,

${ }^{1}$ School of Mathematics, University of New South Wales, Sydney, NSW, 2052, Australia.

(C) Australian Mathematical Society, 1996, Serial-fee code 0334-2700/95 
the motion decays as we move away from the upper surface whereas the motion for the latter decays away from the interface. In most situations, the upper surface is virtually unaffected by the interfacial wave motion. The interfacial waves are shorter and slower than surface waves of the same frequency. When a surface wave encounters a patch of ripples on the bottom, some of its energy will be reflected back and the remainder will propagate beyond the patch. Both the reflected and transmitted parts of the wave motion will in general contain both surface and interfacial wave components. One instructive way of thinking about Bragg effects is in terms of triad resonances between the wavy medium (in this case the bottom corrugations) and two waves travelling through the medium. For an homogeneous fluid, there is only one Bragg resonance - that between the forward- and backward-travelling surface waves. But in a two-layer fluid, there are also possible resonances involving the interfacial waves as well. We will be considering the situation where an incoming surface wave is specified. The additional Bragg resonances of interest will therefore be one involving this wave with a reflected interfacial wave and, perhaps a little surprisingly, another involving the incident wave and a transmitted interfacial wave.

This paper will first describe the two wave modes and then derive the coupled equations which approximately govern the changes in amplitude of the modes as they propagate across small irregularities in an otherwise horizontal bed. Our approach will basically follow that used by Kirby [1] for an homogeneous fluid. Kirby's derivation actually allows the bottom to vary on two lateral scales, one much longer than the wavelength of the waves and another of the same order as the wavelength. The present work could readily be generalised to consider this case. These amplitude equations will then be applied to study the Bragg resonances, both numerically and via a 'modulated wavetrains' approach. Our fundamental finding is that if the incident surface wave is relatively 'long', so that the surface wave 'feels' the bottom and the lower layer is relatively shallow compared with the upper layer, so that the interfacial wave also 'feels' the bottom, then reflected or transmitted interfacial waves of quite large amplitude may occur in the neighbourhood of the appropriate Bragg condition. Although the interface displacements associated with these may be much larger than the displacement of the upper surface caused by the incident surface wave, the amount of energy contained in these interfacial waves is generally quite small. The relevance of these findings to both laboratory and oceanographical situations will then be discussed.

\section{The two wave modes}

The fluid system under consideration consists of two superposed fluids of different densities. We use the subscript 1 to denote quantities in the upper layer, and the 
subscript 2 to denote those in the lower layer. The layers have thicknesses $H_{1}$ and $H_{2}$ and densities $\rho_{1}$ and $\rho_{2}$. The $x$ - and $z$-axes are taken horizontally with the $y$ axis vertically down. The upper surface is at $y=0$ and the bottom at $y=H$, where $H=H_{1}+H_{2}$. Initially, $H_{2}$ will be taken as a constant but will later be allowed to vary. If $p_{0}$ is the constant atmospheric pressure, the pressures in the absence of any motion will be $\mathscr{P}_{1}=p_{0}+\rho_{1} g y$ in the upper layer and $\mathscr{P}_{2}=p_{0}+\rho_{2} g y+g H_{1}\left(\rho_{1}-\rho_{2}\right)$ in the lower layer. We now consider wave motions in which all perturbation quantities are proportional to $\exp (-i \omega t)$ where $\omega>0$. If $p_{1}$ and $p_{2}$ are the perturbation pressures in each layer, and $\eta_{1}$ and $\eta_{2}$ are the displacements of the upper surface and the interface respectively, it is readily shown that the linearised equations are

$$
\nabla^{2} p_{j}=0 \text { for } j=1,2,
$$

subject to

$$
\frac{\partial p_{1}}{\partial y}+\frac{\omega^{2}}{g} p_{1}=0 \quad \text { at } \quad y=0
$$

and

$$
\frac{\partial p_{2}}{\partial y}=0 \quad \text { at } \quad y=H_{1}+H_{2} .
$$

In linear theory, the perturbation pressure in each layer is related to the velocity potential $\phi_{j}$ by $p_{j}=i \omega \rho_{j} \phi_{j}$. The linearised kinematic condition at the interface may be written

$$
\eta_{2}=\frac{1}{\omega^{2} \rho_{j}} \frac{\partial p_{j}}{\partial y} \quad \text { at } \quad y=H_{1} \quad \text { for } \quad j=1,2,
$$

while the linearised condition expressing the continuity of the total pressure across the interface is

$$
p_{1}+\frac{g}{\omega^{2}} \frac{\partial p_{1}}{\partial y}=p_{2}+\frac{g}{\omega^{2}} \frac{\partial p_{2}}{\partial y} \quad \text { at } \quad y=H_{1} .
$$

If we nondimensionalise perturbation pressures with respect to $\rho_{1} g a$, where $a$ is a typical upper-surface displacement due to an incident surface wave, and lengths with respect to $H=H_{1}+H_{2}$, the governing nondimensional equations are now

$$
\begin{aligned}
\nabla^{2} p_{j} & =0 \quad \text { for } j=1,2, \\
\frac{\partial p_{1}}{\partial y}+K p_{1} & =0 \quad \text { at } y=0, \\
\frac{\partial p_{1}}{\partial y} & =\Delta \frac{\partial p_{2}}{\partial y} \quad \text { at } \quad y=D, \\
\frac{\partial p_{1}}{\partial y}+K p_{1} & =\frac{\partial p_{2}}{\partial y}+K p_{2} \quad \text { at } \quad y=D, \\
\frac{\partial p_{2}}{\partial y} & =0 \quad \text { at } y=1,
\end{aligned}
$$


where $D=H_{1} / H, K=\omega^{2} H / g$ and $\Delta=\rho_{1} / \rho_{2}$.

This system has two propagating modes and an infinite number of evanescent ones. The pressure eigenfunction for the propagating modes is given by

$$
\Phi_{1}(y)=\alpha\left(\cosh \tau y-K \tau^{-1} \sinh \tau y\right)
$$

in the upper layer and

$$
\Phi_{2}(y)=\alpha\left(\frac{K \cosh \tau D-\tau \sinh \tau D}{\tau \Delta \sinh \tau(1-D)}\right) \cosh \tau(1-y)
$$

in the lower layer, where $\alpha$ is a constant and $\tau$ satisfies the dispersion relation (see Article 231 of Lamb [2])

$$
\left(\tau^{2}(1-\Delta)+\Delta K^{2}\right) \tanh \tau D \tanh \tau(1-D)-K \tau(\tanh \tau D+\tanh \tau(1-D))+K^{2}=0 .
$$

Without loss of generality, we may take the real roots of this to be positive. There are two of these. The smaller, $\tau_{1}$, is the surface wave root and the larger, $\tau_{2}$, is the interfacial wave root. The subscripts on these refer to the modes, not the layers. It is convenient to choose the constant $\alpha$ to make the dimensionless upper-surface displacement unity for the surface-wave mode $(\alpha=1)$ and the dimensionless interface displacement unity for the interfacial mode $\left(\alpha=\cosh \tau_{2} D-\tau_{2} K^{-1} \sinh \tau_{2} D\right)$. The evanescent modes have purely imaginary values of $\tau$ and will not be considered further.

From now on, the surface wave mode will be indicated by a second subscript 1 and the interfacial wave mode by a second subscript 2 so that $\Phi_{12}$, for example, is the pressure eigenfunction in the upper layer for the interfacial wave mode. The surface and interfacial wave modes are orthogonal in the sense that

$$
\int_{0}^{D} \Phi_{1 k}(y) \Phi_{1 j}(y) d y+\Delta \int_{D}^{1} \Phi_{2 k}(y) \Phi_{2 j}(y) d y=N_{k} \delta_{k j}
$$

The normalisation constants $N_{k}$ are readily found and will not be reproduced here.

\section{Derivation of the amplitude equations}

We now consider the effects of small bottom irregularities on the propagation of the surface and interfacial waves. The bottom is taken to be $y=1+\delta(x, z)$ in dimensionless variables, where $|\delta| \ll 1$. The linearised bottom boundary condition, correct to $\mathrm{O}(\delta)$ may be written

$$
\frac{\partial p_{2}}{\partial y}=\nabla_{2} \cdot\left(\delta \nabla_{2} p_{2}\right) \quad \text { at } \quad y=1,
$$


where $\nabla_{2}$ is the horizontal gradient operator.

The basic assumption to be made is that, because the bottom irregularities are small, the vertical structure of the motion is adequately described by the propagating wave eigenfunctions appropriate to water of constant depth unity. This is thus a straightforward generalisation of the ideas of Kirby [1] to the two-layer case. The evanescent modes are neglected. Thus, we assume that the wave pressure in each layer is given by

$$
p_{j}=\sum_{k=1}^{2} A_{k}(x, z) \Phi_{j k}(y)
$$

The amplitude $A_{k}$ must be the same in each layer because the matching conditions at the interface must hold for all $x$ and $z$.

To investigate how the amplitudes vary, we consider the following integral which is zero because the pressures $p_{j}$ are harmonic:

$$
I_{m}=\int_{0}^{D} \nabla^{2} p_{1} \Phi_{1 m}(y) d y+\Delta \int_{D}^{1} \nabla^{2} p_{2} \Phi_{2 m}(y) d y .
$$

Clearly

$$
I_{m}=\sum_{k=1}^{2} \nabla_{2}^{2} A_{k} N_{k} \delta_{m k}+\int_{0}^{D} \frac{\partial^{2} p_{1}}{\partial y^{2}} \Phi_{1 m}(y) d y+\Delta \int_{D}^{1} \frac{\partial^{2} p_{2}}{\partial y^{2}} \Phi_{2 m}(y) d y .
$$

The terms involving the $y$-derivatives on the right of (4) may be integrated by parts twice. The terms that then arise from the boundary condition at the upper surface and from the interface matching conditions all cancel out and one of the terms from the bottom boundary condition is found to be zero. Thus

$$
I_{m}=N_{m} \nabla_{2}^{2} A_{m}+\int_{0}^{D} p_{1} \frac{d^{2} \Phi_{1 m}}{d y^{2}} d y+\Delta \int_{D}^{1} p_{2} \frac{d^{2} \Phi_{2 m}}{d y^{2}} d y+\Delta\left[\Phi_{2 m} \nabla_{2} \cdot\left(\delta \nabla_{2} p_{2}\right)\right]_{y=1} .
$$

Using (1) and (3), we find that the lateral variations of the mode amplitudes are governed by

$$
\nabla_{2}^{2} A_{m}+\tau_{m}^{2} A_{m}=-S_{m} \sum_{k=1}^{2} P_{k} \nabla_{2} \cdot\left(\delta \nabla_{2} A_{k}\right)
$$

for $m=1,2$, where $P_{k}=\Phi_{2 k}(1)$ and $S_{m}=\Delta N_{m}^{-1} P_{m}$.

Equation (5) can the thought of as a generalisation of (3.8) of Kirby [1] to the case of a two-layer fluid. The two modes are coupled together by the term on the right. 


\section{Bragg resonances}

We consider the situation where the bottom irregularities consist of a patch of sinusoidal corrugations of the form

$$
\delta(x, z)= \begin{cases}\epsilon \sin \lambda x & \text { for }-m \pi / \lambda \leq x \leq 0 \\ 0 & \text { otherwise }\end{cases}
$$

To avoid discontinuities, it will be assumed that $m$ is an integer. A surface wave $A_{1}=\exp \left(-i \tau_{1} x\right)$ is normally incident from $x=+\infty$ and encounters the patch. The solutions for $x \geq 0$ will therefore be

$$
A_{1}=\exp \left(-i \tau_{1} x\right)+R_{S} \exp \left(i \tau_{1} x\right), \quad A_{2}=R_{l} \exp \left(i \tau_{2} x\right),
$$

and for $x \leq-m \pi / \lambda$

$$
A_{1}=T_{S} \exp \left(-i \tau_{1} x\right), \quad A_{2}=T_{1} \exp \left(-i \tau_{2} x\right),
$$

where $R$ and $T$ mean reflection and transmission coefficients respectively and subscripts $S$ and $I$ refer to surface and interfacial waves respectively. Because of the normalisation adopted for the eigenfunctions, $\left|R_{S}\right|$ and $\left|T_{S}\right|$ give the ratios of the maximum upper surface elevation in the reflected and transmitted surface waves to that of the incident wave, whereas $\left|R_{I}\right|$ and $\left|T_{I}\right|$ give the analogous ratios for the displacement of the interface to that of the upper surface due to the incident wave. Energy conservation in this context is

$$
\left|T_{S}\right|^{2}+\left|R_{S}\right|^{2}+\frac{\tau_{2} N_{2}}{\tau_{1} N_{1}}\left(\left|T_{I}\right|^{2}+\left|R_{I}\right|^{2}\right)=1
$$

With the incident surface wave specified, there are three Bragg resonances of relevance.

1. Incident surface wave - reflected surface wave. The Bragg condition for this is $2 \tau_{1}=\lambda$. This problem has been extensively studied for the case of an homogeneous fluid and will not be pursued further here.

2. Incident surface wave - reflected interfacial wave. The Bragg condition for this is $\tau_{1}+\tau_{2}=\lambda$.

3. Incident surface wave - transmitted interfacial wave. The Bragg condition for this is $\tau_{2}-\tau_{1}=\lambda$.

In most situations of oceanographical interest, $\Delta$ is very close to unity which means that $\tau_{2} \gg \tau_{1}$. This, in turn, implies that the last two resonances are very close together. This is illustrated in Figure 1 for $\Delta=0.97, K=0.25, m=20, \epsilon=0.05$ 


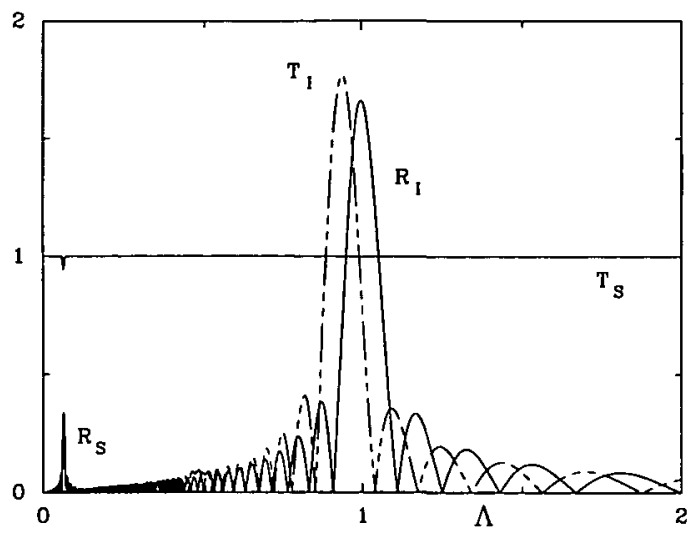

FIGURE 1. The magnitudes of the reflection and transmission coefficients for the surface and interfacial waves as functions of $\Lambda=\lambda /\left(\tau_{1}+\tau_{2}\right)$ in the case $\Delta=0.97, K=0.25, m=20, \epsilon=0.05$ and $D=0.8$.

and $D=0.8$. This density ratio corresponds roughly to the case of fresh water over sea water while the value of $K$ means that the incident surface wave is relatively long and so 'feels' the bottom corrugations. The lower layer is also relatively shallow, so the interfacial wave also 'feels' the bottom corrugations. The abscissa is $\Lambda=\lambda /\left(\tau_{1}+\tau_{2}\right)$ so the resonance of type 2 occurs in the neighbourhood of $\Lambda=1$. This, and the other numerical solutions to be presented later, were obtained by finding the four linearly independent solutions of (5) numerically. Imposing continuity of $A_{1}, A_{2}$ and their derivatives at $x=0$ and $x=-m \pi / \lambda$ enables the reflection and transmission coefficients to be found. Energy conservation was checked numerically. The peak in $\left|R_{S}\right|$ and dip in $\left|T_{S}\right|$ at about $\Lambda=0.06$ represent Bragg reflection of the incident surface wave as a reflected surface wave. Although the peaks in $\left|T_{I}\right|$ and $\left|R_{I}\right|$ appear large, the amount of energy in the interfacial wave motions is quite small, as evidenced by the fact that $\left|T_{S}\right|$ is very close to unity in this region. One lesson from this example is that a small change in the parameters can lead to a large change in the nature of the interfacial wave from large reflection to large transmission.

\section{Modulation solutions}

It is possible to obtain a simplified description of the behaviour of the system near resonance in terms of modulated wavetrains. For the case of an homogeneous fluid, this has been done by Mei [3] and Mei, Hara and Naciri [4] among others.

When the resonance is between an incident surface wave and a reflected interfacial 
wave, we assume that the appropriate Bragg condition is almost satisfied, and so define a detuning parameter $E$ by

$$
\tau_{1}+\tau_{2}=\lambda+2 \epsilon E,
$$

where $E$ measures departures from the Bragg condition. We seek an approximate solution of (5) of the form

$$
\begin{aligned}
& A_{1}=F(\bar{x}) \exp \left(-i \tau_{1} x\right), \\
& A_{2}=B(\bar{x}) \exp \left(i \tau_{2} x\right),
\end{aligned}
$$

where $\bar{x}=\epsilon x$. Substituting these into (5), neglecting all terms of $O\left(\epsilon^{2}\right)$ and above and retaining only the resonant terms in the standard manner leads to

$$
d F / d \bar{x}=\mu_{F} B \exp (2 i E \bar{x})
$$

and

$$
d B / d \bar{x}=\mu_{B} F \exp (-2 i E \bar{x}),
$$

where the interaction coefficients $\mu_{F}$ and $\mu_{B}$ are given by $\mu_{F}=\frac{1}{4} S_{1} P_{2} \tau_{2}$ and $\mu_{B}=$ $\frac{1}{4} S_{2} P_{1} \tau_{1}$. It follows from the definitions of the $S_{k}$ and $P_{k}$ that $\mu_{F} \mu_{B}>0$. The boundary conditions are that $F=1$ at $\bar{x}=0$ (thereby matching to the incident wave) and $B=0$ at $\bar{x}=-m \pi \epsilon / \lambda$ (the radiation condition that there is no incoming wave in $x<-m \pi / \lambda)$. We are interested in $T_{S}=F(-m \pi \epsilon / \lambda)$ and $R_{I}=B(0)$. Equations (8) and (9) are readily solved for $F$ and $B$ subject to the given boundary conditions. We find the following.

1. When $E^{2}<\mu_{F} \mu_{B}$, the case of 'small' detuning

$$
R_{I}=\frac{\mu_{B} \tanh v}{\mu-i E \tanh v}, \quad T_{S}=\frac{\mu \exp (-i m \pi \epsilon E / \lambda)}{\mu \cosh v-i E \sinh v},
$$

where $\mu=\sqrt{\mu_{F} \mu_{B}-E^{2}}$ and $v=m \pi \epsilon \mu / \lambda$.

2. When $E^{2}=\mu_{F} \mu_{B}$, the case of 'critical' detuning

$$
R_{I}=\frac{m \pi \epsilon \mu_{B}}{\lambda-i E m \pi \epsilon}, \quad T_{S}=\frac{\exp (-i m \pi \epsilon E / \lambda)}{\lambda-i E m \pi \epsilon} .
$$

3. When $E^{2}>\mu_{F} \mu_{B}$, the case of 'large' detuning

$$
R_{I}=\frac{\mu_{B} \sin \psi}{\sigma \cos \psi-i E \sin \psi}, \quad T_{S}=\frac{\sigma \exp (-i m \pi \epsilon E / \lambda)}{\sigma \cos \psi-i E \sin \psi},
$$

where $\sigma=\sqrt{E^{2}-\mu_{F} \mu_{B}}$ and $\psi=m \pi \epsilon \sigma / \lambda$. 
Figure 2 shows $\left|R_{l}\right|$ for this modulation theory (broken line) and for the numerical solution (solid line) when $K=0.25, m=20, \Delta=0.97, \epsilon=0.05$ and $D=0.75$. In this example, the transition from small to large detuning occurs at about $\Lambda=$ $1 \pm 0.0005$. This example also illustrates the extreme sensitivity of $R_{I}$ to the depth of the lower layer. Comparing with Figure 1, we see that changing the depth of the lower layer from 0.2 to 0.25 has reduced the maximum value of $\left|R_{I}\right|$ from 1.66 to 0.74 .

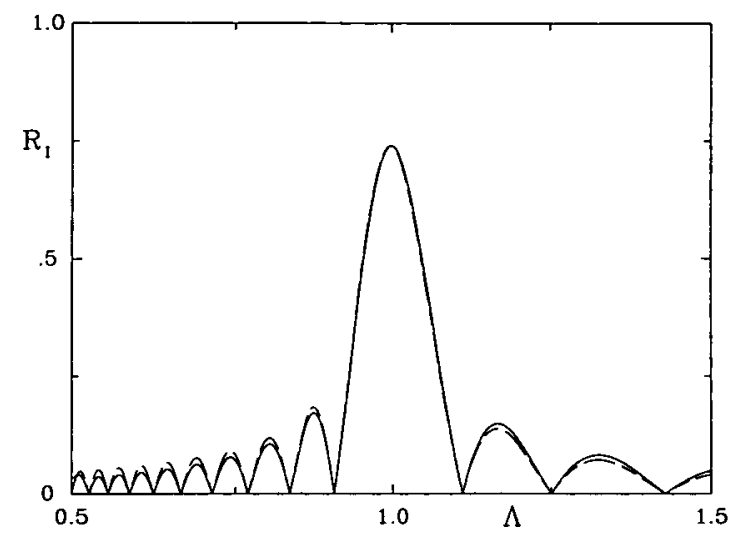

FIGURE 2. The magnitude of the reflection coefficient for the interfacial wave as a function of $\Lambda=\lambda /\left(\tau_{1}+\tau_{2}\right)$ when $\Delta=0.97, K=0.25, m=20, \epsilon=0.05$ and $D=0.75$. The solid line was obtained by numerically integrating (5) and the broken line from the modulation theory.

It is also instructive to ask what happens as $m \rightarrow \infty$, that is, when the incident surface wave encounters a semi-infinite patch of corrugations. In the cases of small detuning and critical detuning, it is readily shown from the above expressions that $T_{S} \rightarrow 0$ and $\left|R_{I}\right| \rightarrow \sqrt{\mu_{F} / \mu_{B}}$. In these cases, all the incident surface wave energy is reflected back as an interfacial wave. For the case of large detuning this is not so and $\left|R_{I}\right|$ has no limiting value as $m \rightarrow \infty$ and can lie anywhere between 0 and $\left|\mu_{B} / E\right|$.

When the resonance is between an incident surface wave and a transmitted interfacial wave, the analysis is similar except that the detuning parameter is now defined by $\tau_{2}-\tau_{1}=\lambda-2 \epsilon E$. Then with

$$
\begin{aligned}
& A_{1}=F(\bar{x}) \exp \left(-i \tau_{1} x\right), \\
& A_{2}=B(\bar{x}) \exp \left(-i \tau_{2} x\right),
\end{aligned}
$$

we again derive (8) and (9), except that the interaction coefficient $\mu_{B}$ is now given by $\mu_{B}=-\frac{1}{4} S_{2} P_{1} \tau_{1}$. In this case $\mu_{F} \mu_{B}<0$ and the boundary conditions are $F=1$ and $B=0$ at $\bar{x}=0$. We find

$$
\left.T_{I}=B(-m \pi \epsilon / \lambda)\right)=-\left(\mu_{B} / \nu\right) \sin (m \pi \epsilon \nu / \lambda) \exp (i m \pi \epsilon E / \lambda),
$$




$$
\left.T_{S}=F(-m \pi \epsilon / \lambda)\right)=[\cos (m \pi \epsilon \nu / \lambda)-(i E / \nu) \sin (m \pi \epsilon \nu / \lambda)] \exp (-i m \pi \epsilon E / \lambda),
$$

where $\nu=\sqrt{E^{2}-\mu_{F} \mu_{B}}$.

At perfect tuning $(E=0)$, it is clear from these expressions that it is possible for some parameter values for $\left|T_{S}\right|$ to be zero. In this instance, all the energy of the incoming surface wave is transferred to the transmitted interfacial wave. Neither $\left|T_{I}\right|$ nor $\left|T_{S}\right|$ has any limit as $m \rightarrow \infty$. Some results for $\left|T_{I}\right|$ when $\Delta=0.8, K=0.5$, $m=10, D=0.6$ and $\epsilon=0.1$ are shown in Figure 3. The modulation theory results are shown by a broken line and the numerical solution by a solid line. The density ratio corresponds roughly to a laboratory situation of kerosene over water. The abscissa is $\Gamma=\lambda /\left(\tau_{2}-\tau_{1}\right)$ and hence the Bragg condition here is satisfied at $\Gamma=1$.

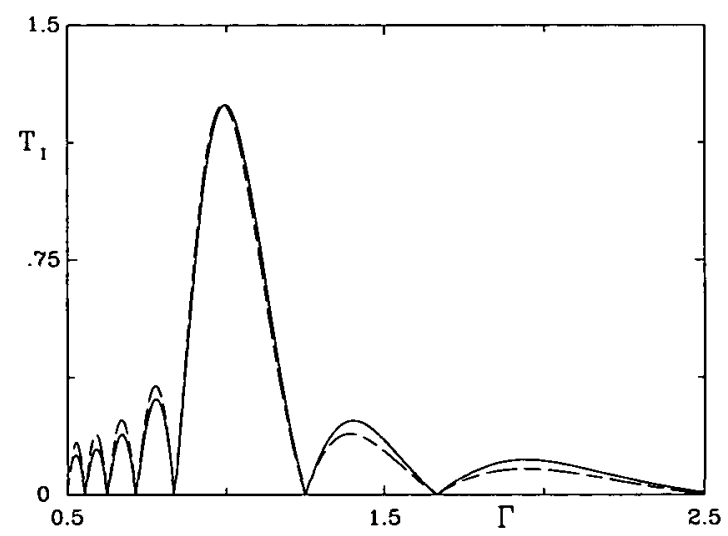

FIGURE 3. The magnitude of the transmission coefficient for the interfacial wave as a function of $\Gamma=\lambda /\left(\tau_{2}-\tau_{1}\right)$ when $\Delta=0.80, K=0.5, m=10, \epsilon=0.1$ and $D=0.6$. The solid line was obtained by numerically integrating (5) and the broken line from the modulation theory.

\section{Further numerical results and discussion}

The mechanism discussed in this paper provides a means for generating very short interfacial waves at surface-wave frequencies. It is clear that the prerequisites for relatively large amplitude reflected or transmitted interfacial waves to be produced from an incident surface wave by Bragg resonance effects with bottom corrugations is that the incident surface wave be relatively long and the lower layer be relatively thin compared with the upper layer. These points are illustrated in Figures 4 and 5 which show contours of $\left|R_{l}\right|$ as functions of $\Lambda$ and $K$ or $D$. Results for $\left|T_{l}\right|$ as a function of $\Gamma$ are broadly similar and will not be reproduced. In Figure $4, \Delta=0.97, m=15$, $\epsilon=0.05$ and $D=0.8$. In Figure $5, \Delta=0.8, m=15, \epsilon=0.05$ and $K=0.75$. In 
the latter case, the incident wave is not particularly 'long' so the reflection is relatively weak.

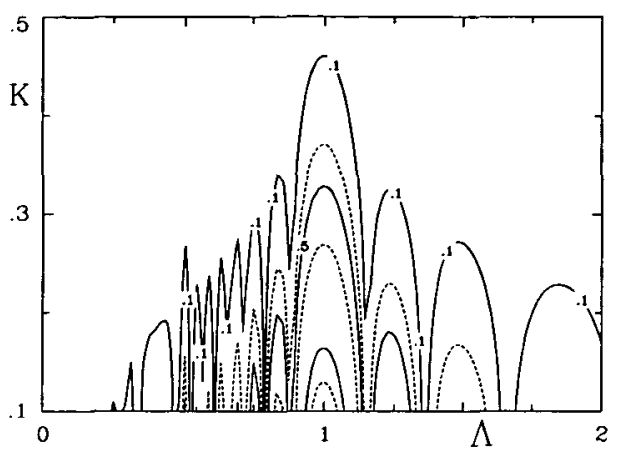

FIGURE 4. Contours of the magnitude of the reflection coefficient for the interfacial wave as a function of $K$ and $\Lambda$ when $\Delta=0.97, m=15, \epsilon=0.05$ and $D=0.8$. The contour levels are $\left|R_{l}\right|=0.1,0.3,0.5,1.0,3.0$ and 4.0 .

The question now arises as to the relevance of these results and the situations where the Bragg effects discussed here might be expected to be of importance. Reverting to dimensional variables, if $T$ is the wave period in seconds, then $H=K g T^{2} / 4 \pi^{2} \simeq$ $K T^{2} / 4$ metres. For the parameters used in Figure 3, a wave period of 2 seconds corresponds to a depth $H=0.5$ metres. The two dimensionless wavenumbers are $\tau_{1}=0.7876$ and $\tau_{2}=4.6438$ approximately. The bottom wavelengths required for Bragg effects to produce a large reflected or transmitted wave are then approximately $\pi /\left(\tau_{2} \pm \tau_{1}\right)$ metres, that is, 0.587 metres and 0.814 metres respectively. These are both entirely reasonable figures for a laboratory situation. For the more oceanographically relevant cases depicted in Figure 1, the values $\tau_{1}$ and $\tau_{2}$ are 0.52295 and 16.43992 respectively. A wave period of $T=8$ seconds and total water depth of $H=4$ metres gives $K=0.25$ approximately. The appropriate bottom corrugation wavelengths for the Bragg resonances are then $8 \pi /\left(\tau_{2} \pm \tau_{1}\right)$ metres, that is, 1.48 and 1.58 metres respectively. Again, these are reasonable figures. The requirement for the bottom layer to be relatively thin would occur, for example, in the situation where salty water intrudes under fresh water.

The extension of this work to obliquely-incident waves is immediate. If $\theta$ is the angle of incidence, the $z$ wavenumber of both waves will be $n=\tau_{1} \sin \theta$. The $x$ wavenumber of the surface wave mode is then $\sigma_{1}=\tau_{1} \cos \theta$ and that for the interfacial wave mode is $\sigma_{2}=\sqrt{\tau_{2}^{2}-n^{2}}$. In the Bragg conditions, $\sigma_{i}$ replaces $\tau_{i}$. There are corresponding changes to all the relevant formulae, the most important being that, in the modulation theory expressions for $\mu_{F}$ and $\mu_{B}, \tau_{1}$ is replaced by $\sigma_{1}-n^{2} / \sigma_{2}$ and $\tau_{2}$ by $\sigma_{2}-n^{2} / \sigma_{1}$. It is worth noting that, because $\tau_{2}$ is generally much greater than $\tau_{1}, \sigma_{2}$ 


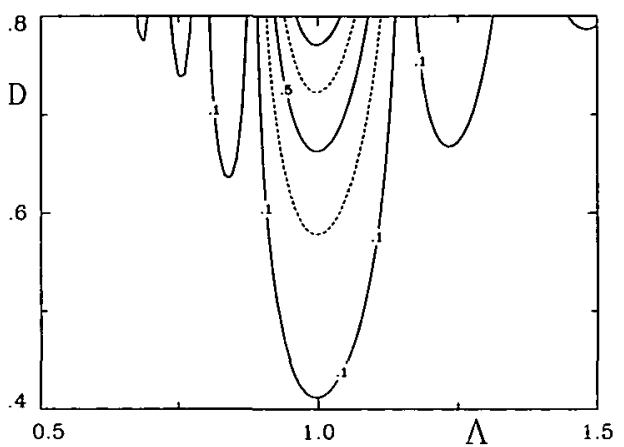

FIGURE 5. Contours of the magnitude of the reflection coefficient for the interfacial wave as a function of $D$ and $\Lambda$ when $\Delta=0.80, m=15, \epsilon=0.05$ and $K=0.75$. The contour levels are $\left|R_{I}\right|=0.1,0.3,0.5,0.7,0.9$ and 1.1 .

will be relatively insensitive to changes in $\theta$ so the transmitted and reflected interfacial waves will have their crests almost parallel to the bottom contours, irrespective of the angle of incidence of the surface wave.

\section{Acknowledgements}

This work was performed at the Department of Mathematics, Monash University while the author was on study leave from the University of New South Wales. He thanks both institutions for making this possible.

\section{References}

[1] J. T. Kirby, “A general wave equation for waves over rippled beds ", J. Fluid Mech. 162 (1986) 171-186.

[2] H. Lamb, Hydrodynamics (Cambridge University Press, 1932).

[3] C. C. Mei, "Resonant reflection of surface water waves by periodic sandbars", J. Fluid Mech. 152 (1985) 315-335.

[4] C. C. Mei, T. Hara and M. Naciri, "Note on Bragg scattering of water waves by parallel bars on the seabed ", J. Fluid Mech. 186 (1988) 147-162.

[5] T. J. O'Hare and A. G. Davies, "A comparison of two models for surface-wave propagation over rapidly varying topography ", Applied Ocean Research 15 (1993) 1-11. 